\title{
Asteroseismic modelling of Procyon A: Preliminary results
}

\author{
G. Doğan $^{1, \star}$ A. Bonanno ${ }^{2}$ T. R. Bedding ${ }^{3}$ T. L. Campante ${ }^{1,4}$ J. Christensen-Dalsgaard ${ }^{1}$ \\ and H. Kjeldsen ${ }^{1}$ \\ 1 Department of Physics and Astronomy, Aarhus University, Ny Munkegade, DK-8000, Aarhus C, Denmark \\ 2 Catania Astrophysical Observatory, Via S.Sofia 78, 95123, Catania, Italy \\ 3 School of Physics A29, University of Sydney, NSW 2006, Sydney, Australia \\ ${ }^{4}$ Centro de Astrofísica da Universidade do Porto, Rua das Estrelas, 4150-762 Porto, Portugal
}

Received XX, accepted XX

Key words stars: individual (Procyon A) - stars: oscillations - stars: evolution

We present our preliminary results of the modelling of the F5 star Procyon A. The frequencies predicted by our models are compared with the frequencies extracted through a global fit to the power spectrum obtained by the latest ground-based observations, which provides two different mode identification scenarios.

(C) 2010 WILEY-VCH Verlag GmbH \& Co. KGaA, Weinheim

\section{Introduction}

Procyon $\mathrm{A}$ is a member of a binary system with a white dwarf companion, Procyon B. It is one of the very bright stars to the naked eye, and hence it has been very attractive for the observers. The observational constraints which we adopted are summarized in Section 2. It has also been of asteroseismic interest for a long while (see Arentoft et al. 2008, for a summary), with a solar-like power excess in its spectrum first reported by Brown et al. (1991). However, there has been no agreement on the individual oscillation frequencies. Several authors have investigated the structure and evolution of Procyon A through an asteroseismic approach (e.g., Guenther \& Demarque 1993; Barban et al. 1999; Chaboyer et al. 1999; Di Mauro \& Christensen-Dalsgaard 2001; Eggenberger et al. 2005; Provost et al. 2006, Bonanno et al. 2007), but there has been a need for more accurate frequencies. Recently, the star was observed through a multi-site campaign by eleven telescopes for more than three weeks (Arentoft et al. 2008). The frequency analysis is described by Bedding et al. (2010). They presented results from different approaches of frequency extraction: Iterative sine-wave fitting and global fitting to the power spectrum. In the former method, a sine wave is fitted to each mode one after the other while the corresponding sinusoid is subtracted from the time series at each step. This is repeated until the signal to noise ratio of the remaining power is lower than a given threshold. This method was used for frequency extraction of groundbased radial velocity data before (see, e.g., the analysis on solar-like star $\beta$ Hyi by Bedding et al. (2007)). In the latter method, the goal is to find an overall fit to the power spectrum for all the frequencies, mode heights, and linewidths simultaneously, using some prior knowledge of oscillation

\footnotetext{
^ e-mail: gulnur@phys.au.dk
}

properties as constraints. A similar implementation of this method was previously applied to space-based data (see, e.g., frequency analysis of CoRoT star HD 49933 by Benomar et al. (2009)). In this work, we adopted this Bayesian approach which provided us with two mode identification scenarios, referred to as Scenario A and B, with different posterior odds (for a detailed discussion, see Bedding et al. 2010). We chose the output of this analysis in order to test both scenarios. Note that Scenario B was favoured by Bedding et al. (2010; see that paper for a discussion), but here we test both scenarios.

\section{Methods and Tools}

We have adopted the following properties for the position of the star in the H-R Diagram: $T_{\text {eff }}=6530 \pm 90 \mathrm{~K}$ (Fuhrmann et al. 1997) and $\log \left(L / L_{\odot}\right)=0.85 \pm 0.06$ (Steffen 1985). We note that there are several revised values for luminosity in the literature, such as $\log \left(L / L_{\odot}\right)=0.840 \pm 0.018$ derived by Jerzykiewicz \& Molenda-Żakowicz (2000) using the Hipparcos parallax and total absolute flux; however, we chose to scan a wider range, which largely covers most of the revised values. A similar argument applies also to the choice of the effective temperature. We have not put an additional constraint on the radius for the time being, although we do compare the stellar mean density inferred from the analysis with the value $0.172 \pm 0.005 \rho_{\odot}$, obtained from the measured radius using the angular diameter $5.404 \pm 0.031$ mas (Aufdenberg et al. 2005) and the revised Hipparcos parallax (284.56 \pm 1.26 mas 1 , van Leeuwen 2007), together with the adopted mass $1.463 \pm 0.033 M_{\odot}$, which is the mean of the two different astrometric determinations (Girard et al. 2000,

\footnotetext{
${ }^{1}$ Note that there is an error in Bedding et al. 2010 (Section 9); the value they give for the revised parallax is actually the original one. We also note that the uncertainty on the revised parallax is larger than the original but is presumably more reliable.
} 
Gatewood and Han 2006). For the metallicity of the star, we allowed a wide range covering the 0.05 dex iron deficiency suggested by Allende Prieto et al. (2002), and we used the solar mixture from Grevesse \& Noels (1993).

We have computed stellar models with two different evolutionary codes: ASTEC (Aarhus STellar Evolution Code) (Christensen-Dalsgaard 2008a) and GARSTEC (Garching Stellar Evolution Code) (Weiss \& Schlattl 2008). The method we used is to compute several grids of standard models scanning through a parameter space formed by varying the mass, the initial metallicity at the stellar surface, $Z_{\mathrm{i}} / X_{\mathrm{i}}$, and the mixing-length parameter, $\alpha$, where the mixing length is defined as $\ell=\alpha H_{p}, H_{p}$ being the pressure scale height. So far, we have searched within the following ranges: $M=1.42-1.52 M_{\odot}, Z_{\mathrm{i}} / X_{\mathrm{i}}=0.0204-0.0245$, $Y_{\mathrm{i}}=0.26-0.31$, and $\alpha=1.6-1.9$. Here $X_{\mathrm{i}}, Y_{\mathrm{i}}$, and $Z_{\mathrm{i}}$, are the initial mass fractions of hydrogen, helium, and the elements heavier than helium, respectively. We have computed the models without taking into account diffusion, convective overshooting, or rotation.

The Aarhus adiabatic pulsation package (ADIPLS) (Christensen-Dalsgaard 2008b) has been used to calculate the frequencies of the models having properties that are in agreement with the observations. We have then compared the model frequencies with the observed frequencies. We selected the models that minimize the following $\chi^{2}$ :

$$
\chi^{2}=\sum_{n, l}\left(\frac{\nu_{l}^{\mathrm{obs}}(n)-\nu_{l}^{\mathrm{model}}(n)}{\sigma\left(\nu_{l}^{\mathrm{obs}}(n)\right)}\right)^{2},
$$

where $\nu_{l}^{\mathrm{obs}}(n)$, and $\nu_{l}^{\operatorname{model}}(n)$ are the model, and the observed, frequencies with spherical degree $l$ and radial order $n$, and $\sigma\left(\nu_{l}^{\text {obs }}(n)\right)$ represents the uncertainties in the observed frequencies.

\section{Results}

The results from the two different stellar evolution codes are similar; hence we present some of the selected models computed with ASTEC. The so-called échelle diagrams of the best models for both of the scenarios, chosen without applying any near-surface corrections are shown in Figs 1 and 2, with their parameters summarized in Table 1. One plots the échelle diagrams using the frequency modulo the large frequency separation, $\Delta \nu$, in the horizontal axis. In order to allow easy comparison with the diagrams shown by Bedding et al. (2010), we use the same value, $\Delta \nu=56 \mu \mathrm{Hz}$, in our diagrams.

The behaviour of the frequency differences between the models and the observations (shown in Figs 3 and 4) are quite different from that in the Sun. Kjeldsen et al. (2008) showed that the difference between observed and model frequencies of the Sun can be fitted by a power law, which can also be employed to correct the model frequencies for nearsurface effects in other solar-like stars, such as $\beta$ Hyi and

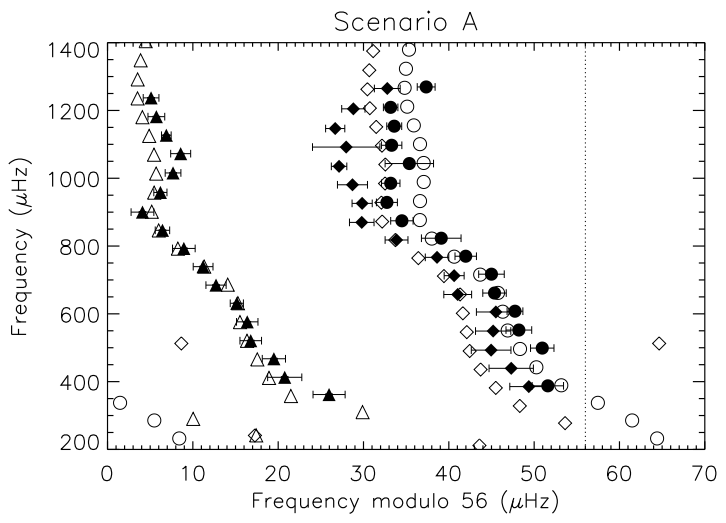

Fig. 1 Échelle diagram of the selected model for Scenario A. Open symbols represent the model frequencies, while the filled symbols with the uncertainties correspond to the frequencies extracted from the observations. Circles, triangles, and diamonds are used for the modes with spherical degree $l=0,1$, and 2 , respectively. The vertical dot-dashed line corresponds to the value of $\Delta \nu$.

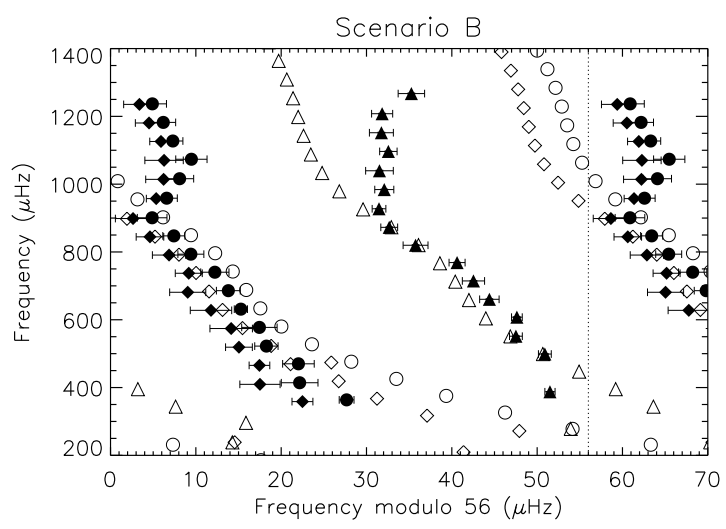

Fig. 2 Échelle diagram of the selected model for Scenario B. Symbols are used in the same way as in Fig. 1.

$\alpha$ Cen A. However, in Procyon, we cannot justify the application of such a surface correction to yield a significant improvement in the fit, since the frequency differences do not follow the power-law behaviour (see also Figs 16 and 17 of Bedding et al. 2010).

\section{Discussion and Conclusion}

We can argue, if Scenario A is the correct one, that the predictions of the stellar evolutionary models match the observations quite well; however, a surface correction for the model frequencies seems not to be needed, unlike in the solar case. Therefore, the effects of different near-surface properties on the high frequencies might be cancelling out in Procyon; this deserves further investigation.

If, on the other hand, Scenario B is correct, there seems to be no good agreement between the models and the ob- 
Table 1 Parameters of the best models

\begin{tabular}{c|c|c}
\hline Parameter & Scenario A & Scenario B \\
\hline$M / M_{\odot}$ & 1.50 & 1.50 \\
$Z_{\mathrm{i}} / X_{\mathrm{i}}$ & 0.0235 & 0.0245 \\
$Y_{\mathrm{i}}$ & 0.266 & 0.290 \\
Age $(\mathrm{Gyr})$ & 1.83 & 1.51 \\
$R / R_{\odot}$ & 2.058 & 2.067 \\
$\rho / \rho_{\odot}$ & 0.1721 & 0.1698 \\
$L / L_{\odot}$ & 6.565 & 7.286 \\
$T_{\text {eff }}(\mathrm{K})$ & 6446 & 6603 \\
$\alpha$ & 1.8 & 1.6 \\
$X_{c}^{*}$ & 0.1585 & 0.1995 \\
\hline$\chi^{2}$ & 3.79 & 24.46 \\
\hline
\end{tabular}

* Mass fraction of hydrogen remaining in the centre of the star

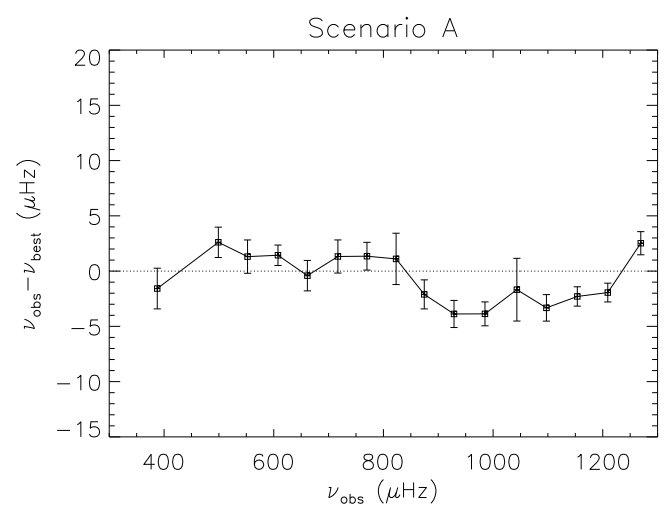

Fig. 3 The difference between the radial $(l=0)$ frequencies from the observations and the selected model for Scenario A. The indicated uncertainties are those from the data analysis.

servations, even in the low-frequency region, which means that there is something incompatible in the structure of the models.

We have presented preliminary results from our ongoing work. To come to a conclusion we need to extend our analysis. Effects of inclusion of overshooting and use of different treatments of convection should be analysed. In addition, the suspected mixed mode reported by Bedding et al. (2010) could help us distinguish between the two scenarios, and put further constraints on the age, and the chemical composition.

Although from the point of view of modelling, Scenario A seems to be less problematic, we cannot yet strongly favour either of the scenarios; therefore, it is difficult to set accurate constraints on the stellar properties. Nevertheless, either of the cases suggests that Procyon is quite different from the Sun, which provides a very good opportunity to test our understanding of stellar structure and evolution.

Acknowledgements. This work was supported by the European Helio- and Asteroseismology Network (HELAS), a major international collaboration funded by the European Commission's Sixth Framework Programme. GD, JC-D, and HK acknowledge financial support from the Danish Natural Science Research Council.

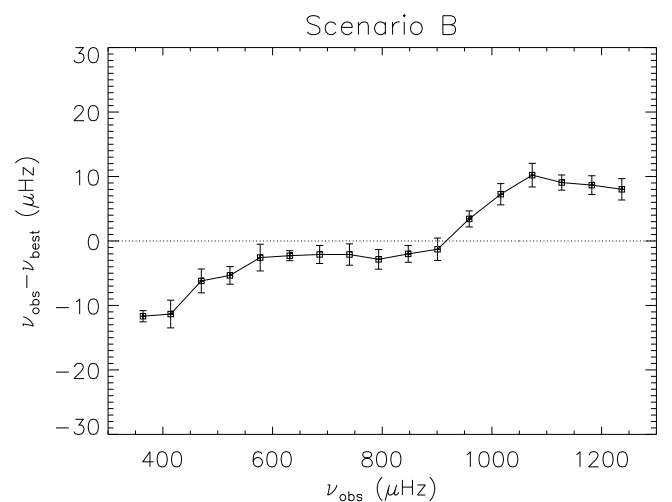

Fig. 4 Same as Fig. 3, but for Scenario B

GD would like to thank Pierre-Olivier Quirion for providing a parallel-programming code for faster evolution computation.

\section{References}

Allende Prieto, C., Asplund, M., López, R. J. G. \& Lambert, D. L.: 2002, ApJ 567, 544

Arentoft, T., Kjeldsen, H., Bedding, T. R. et al.: 2008, ApJ 687, 1180

Aufdenberg, J.P., Ludwig, H.-G. \& Kervella, P.: 2005, ApJ 633, 424

Barban, C., Michel, E., Martic, M. et al.: 1999, A\&A 350, 617

Bedding, T. R., Kjeldsen, H., Arentoft, T. et al.: 2007, ApJ 663, 1315

Bedding, T. R., Kjeldsen, H., Campante, T. L. et al.: 2010, ApJ 713, 935

Benomar, O., Appourchaux, T. \& Baudin, F.: 2009, A\&A 506, 15

Bonanno, A., Küker, M. \& Paternò, L.: 2007, A\&A 462, 1031

Brown, T. M., Gilliland, R. L., Noyes, R. W. \& Ramsey, L. W.: 1991, ApJ 368, 599

Chaboyer, B., Demarque, P. \& Guenther, D. B.: 1999, ApJ 525, L41

Christensen-Dalsgaard, J.: 2008a, Ap\&SS 316, 13

Christensen-Dalsgaard, J.: 2008b, Ap\&SS 316, 113

Di Mauro, M. P. \& Christensen-Dalsgaard, J.: 2001, IAUS 203, 94

Eggenberger, P., Carrier, F. \& Bouchy, F.: 2005, New Astron. 10, 195

Fuhrmann, K., Pfeiffer, M., Franck, C. et al.: 1997, A\&A 323, 909

Gatewood, G. \& Han, I.: 2006, AJ 131, 1015

Girard, T. M. et al.: 2000, AJ 119, 2428

Grevesse, N. \& Noels, A.: 1993, in Origin and Evolution of the Elements, edited by N. Prantzos, E. Vangioni-Flam \& M. Cassé, (Cambridge: Cambridge Univ. Press), p. 15

Guenther, D. B. \& Demarque, P.: 1993, ApJ 405, 298

Jerzykiewicz, M. \& Molenda-Żakowicz, J.: 2000, Acta Astronomica 50,369

Kjeldsen, H., Bedding, T. R. \& Christensen-Dalsgaard, J.: 2008, ApJ 683, L175

Provost, J., Berthomieu, G., Martić, M. \& Morel, P.: 2006, A\&A 460,759

Steffen, M.: 1985, A\&AS 59, 403

van Leeuwen, F.: 2007, Hipparcos, the New Reduction of the Raw Data, Astrophys. Space Sci. Library, 350

Weiss, A. \& Schlattl, H.: 2008, Ap\&SS 316, 99 